\title{
COMBINATION OF A FAST WHITE-LIGHT INTERFEROMETER WITH A PHASE SHIFTING INTERFEROMETRIC LINE SENSOR FOR FORM MEASUREMENTS OF PRECISION COMPONENTS
}

\author{
Jörg Riebeling ${ }^{1}$, Peter Lehmann ${ }^{1}$, Sören Laubach ${ }^{2}$, Gerd Ehret ${ }^{2}$ \\ ${ }^{1}$ Universität Kassel, Wilhelmshöher Allee 71, 34121 Kassel, Germany; \\ ${ }^{2}$ Physikalisch-Technische Bundesanstalt, Bundesallee 100, 38116 Braunschweig, Germany.
}

\begin{abstract}
Modern industrial fabrication processes put high requirements on the quality of the surface form of precision-machined components, e.g. optical lenses or microelectromechanical systems (MEMS). Optical sensors provide high precision non-contact surface measurement to verify these quality requirements, even on fragile surfaces.

The low-cost line-scanning interferometer that is presented in this contribution is based on a Michelson interferometer configuration in combination with a high-speed line-scan camera. The sensor can operate in scanning white-light or in optical path length modulation (OPLM) mode. The white-light mode is used to automatically align the sensor perpendicular in the working distance of $13 \mathrm{~mm}$ to the surface of the specimen. In OPLM-mode, an oscillating reference mirror and a band-pass filtered light source are used, to measure the form of a radial symmetric specimen with a diameter of up to $25.4 \mathrm{~mm}$ with interferometric accuracy. Several overlapping ring-shaped sub-apertures are measured iteratively in different radial positions until the whole surface is scanned. The sub-apertures are stitched together to reconstruct the complete 3D-topography, while overlapping areas can be used to correct movement errors of the scanning axes. This concept is highly adaptive and can be applied to many different specimen geometries e.g. planes, spheres or aspheric glass lenses.
\end{abstract}

Keywords: interferometry, optical form measurement, aspheric lens measurement

\section{INTRODUCTION}

As a result of improved precision machining methods like diamond turning or ion beam processing, more precise mechanical components or optical surfaces can be produced. Accurate form measurement is one of the key technologies to verify the quality of those surfaces. Optical sensors for example are based on different approaches [1] but can roughly be divided into pointwise and areal methods. Pointwise sensors are moved laterally along a specimen's surface to obtain a $3 \mathrm{D}$ point cloud. Therefore, measuring a complete $3 \mathrm{D}$ topography is time-consuming and the accuracy of the sensor is mainly influenced by the accuracy of its movement system. Point sensors can be easily adapted to different specimen geometries as they have large measurement ranges and workings distances. Areal surface sensors are using optical detectors that measure more than one surface position simultaneously. This reduces the time to scan a complete specimen's surface but increases the effort that has to be done for alignment. Due to a restricted measurement range of the optical system, large surface-height variations within the measurement area can only be measured by using additional mechanical or optical elements (e.g. computer-generated holograms [2] or optical path length variation). Commonly used methods are scanning white-light (SWLI) or phase-shifting interferometry (PSI) [3], [4]. Using 
SWLI, a depth scan is performed by moving a specimen along the optical axis. Due to the high spectral bandwidth of the illuminating white-light source, interference fringes appear when the length of the optical paths between the reference and the test beam differs by not more than half of the coherence length of the light. In contrast to this, a phase-shifting interferometer uses a light source with a smaller spectral bandwidth, e.g. a laser diode or a band-pass filtered LED. The phase of the interference signal can be shifted by modulating the optical path length of either the reference or the measurement path in defined steps of fractions lower than a quarter of the lights centre wavelength. The phase can also be shifted by using optical polarizers, and the interferogram can be recorded in a single image frame [5]. If the whole surface of the specimen cannot be captured by one image, stitching methods can be used to combine several sub-apertures to form a complete topography. Commercial systems which use stitching technologies are available [6].

In contrast to existing approaches, the sensor that is presented in this contribution uses a linescan sensor that is tracked to the specimen's contour [7], [8], [9]. The detector is combined with a Michelson interferometer setup that is capable to obtain height values with interferometric accuracy. The line sensor combines the advantages of areal scanning methods like fast scanning speeds with those of pointwise sensors e.g. minor restrictions on the surface under test. A fast oscillating reference surface is used to sinusoidally modulate the optical path length of the reference path (Sasaki et al. [10], [11]) which allows the sensor to achieve high measurement speeds [12], [13]. This paper presents the current setup of a measurement system that combines SWLI and PSI by OPLM. The calibration and measurement strategies are shown, and some example measurements regarding the repeatability of the sensor are presented. Finally, some possible improvements to the system are given.

\section{SENSOR SETUP}

The line-scanning sensor, as depicted in Figure 1, is based on a Michelson interferometer configuration, which is mounted in front of an apochromatic long working distance microscope objective with a 5x magnification. The Sensor uses two different light sources for its operation modes. A broadband white-light LED with a coherence length of about $2 \mu \mathrm{m}$ is used as light source for the white-light scanning mode, increasing the accuracy of the height evaluation of the scan. A band-pass filtered green LED is used as light source for the sinusoidal phase-shifting mode. The filter reduces the bandwidth of the light source (center wavelength: $532 \mathrm{~nm}$, Full width at half maximum: $10 \mathrm{~nm}$ ), thereby increasing the coherence length and the measurement range along the optical axis of the sensor to about $18 \mu \mathrm{m}$. To avoid mechanical vibrations in case of switching between the measuring modes, the light of the LEDs is guided by an optical beam splitter onto the specimen instead by a movable mirror. A highspeed line-scan camera, that is capable to record images with a frame rate of $54.000 \mathrm{fps}$, is used as detector. The camera is positioned with respect to the radial direction of the specimen in the $\mathrm{x}$-direction. In white-light mode, the camera is triggered by the scanning axis that allows the sensor to perform a white-light interferometric height scan while the scanning axis is moving with a speed of several $\mathrm{mm}$ per second. In OPLM-mode, a signal generation device is used to trigger the camera synchronized to the actors excitation signal, offering a measurement rate of approximately 400 height values per second for each camera pixel. Using the presented components, the sensor has a field of view of approx. $2 \mathrm{~mm} \times 2 \mu \mathrm{m}$. Due to the limited coherence length, the magnification of the microscope objective and the detectors cell size, the setup is capable to measure surfaces with a minimum radius of curvature of about $50 \mathrm{~mm}$.

The sensor is moved by a five-axis motion system (axes $\mathrm{x}, \mathrm{y}, \mathrm{z}, \mathrm{x}_{\mathrm{b}}$ and $\mathrm{b}$ ) and the specimen is mounted in the center of a rotational table (axis c) underneath the sensor. A manual tilt-stage 
and a xy-stage is mounted onto the c-axis to align the specimen with respect to the rotational axis c. The y-axis is used to align the sensor to the center of rotation of the c-axis.

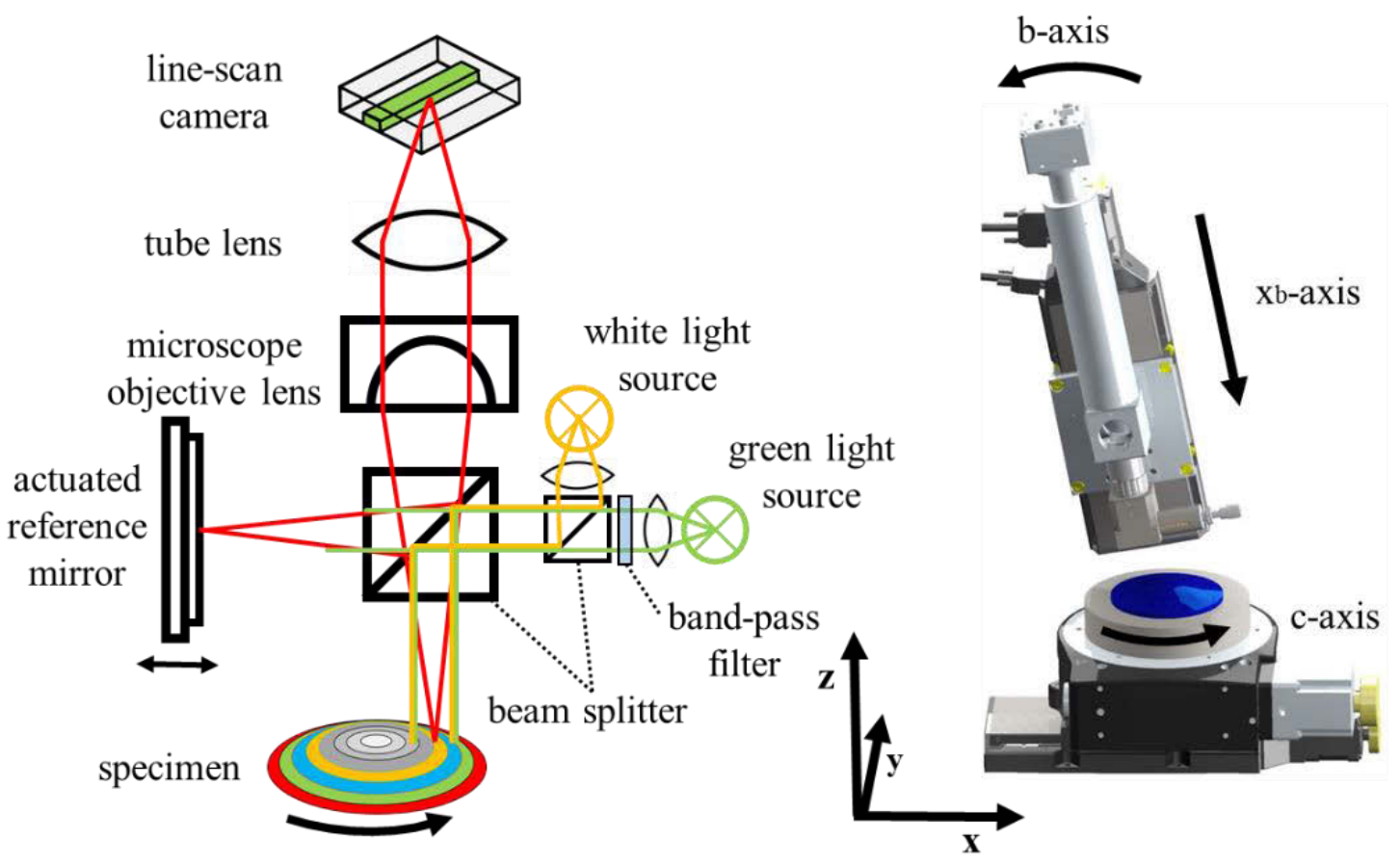

Figure 1: left: schematic diagram of the line-scan Michelson interferometer with white-light and optical path length modulation operation mode, red: imaging path, green/yellow: illumination path; right: model of the implemented setup showing the interferometric sensor and the simplified positioning system.

The white-light scan is performed by a high-precision linear axis (axis $\mathrm{x}_{\mathrm{b}}$ ) that comprises a linear encoder with a calculated resolution of $1 \mathrm{~nm}$ and a repeatability of $50 \mathrm{~nm}$. The OPLMmode scan is performed by the rotational axis c, using a typical scanning speed of $10 \% \mathrm{~s}$, the measurement time to measure one sub-aperture is about half a minute, the current evaluation algorithm takes a few minutes.

\section{CALIBRATION OF THE SETUP}

Due to fabrication variations and mechanical deformation, the setup is calibrated before a measurement is done. This calibration comprises the characterization of the reference mirrors movement as well as the detection of its geometry. Both can be done measuring a flat surface standard in OPLM-mode for a few seconds while the sensor is not moving. The major fringefrequency of the obtained interference signal from each pixel is calculated and stored. This frequency depends on the actor's local oscillation amplitude that varies due to deformation of the actor's surface during movement. Further evaluation processes use the stored frequency, as the phase component of the obtained signal is calculated just for this frequency (lock-in detection). This drastically reduces the effort of the evaluation algorithm and making it more robust to bad quality signals. The calibration measurement data is evaluated, and the mean height of each pixel is stored describing the surface height profile of the reference mirror. The topography of the reference mirror is subtracted from each evaluated height profile afterwards. 


\section{MEASUREMENT STRATEGY}

Prior to the measurement of one ring-shaped sub-aperture, the sensor is automatically tracked to the specimen's topography by an interferometric white-light scan. Therefore, the white light source is switched on and the sensor performs a depth-scan with a speed of up to $5 \mathrm{~mm} / \mathrm{s}$ using the $\mathrm{x}_{\mathrm{b}}$-axis. The mean height and the slope of the specimen's surface are measured and the sensor is positioned perpendicular and in working distance to the surface under test. After the tracking is finished, the sensor switches to OPLM-mode by changing the illuminating light source and activating the oscillating reference mirror whereby the phase of the interference signal is modulated periodically. The surface scan starts by activating the external camera trigger device that emits trigger pulses that are synchronized to the reference mirrors excitation signal. While the camera is triggered, the specimen is rotated by $370^{\circ}$ as depicted in Figure 2 , using the c-axis at a speed of about $10^{\circ} / \mathrm{s}$. The redundant data from the $10^{\circ}$ overlap can be used to confirm or correct the obtained data, depending on the quality of the used movement system. After one sub-aperture is measured, the sensor is moved by half of its field of view, which is about $1 \mathrm{~mm}$ with the currently used microscope objective lens, in x-direction and the tracking process is repeated. The resulting overlap in x-direction is used to stitch two sequenced subapertures. Each ring-shaped sub-aperture that is measured consist of about 14.000 height values per pixel of the line-scan camera. Measuring the surface of the circumference of a specimen with a diameter of $25.4 \mathrm{~mm}$ at a speed of $10^{\circ} / \mathrm{s}$ results in a spatial measurement interval of 5.6 $\mu \mathrm{m}$, the estimated lateral resolution of the system using the Rayleigh criterion is about $2.3 \mu \mathrm{m}$.

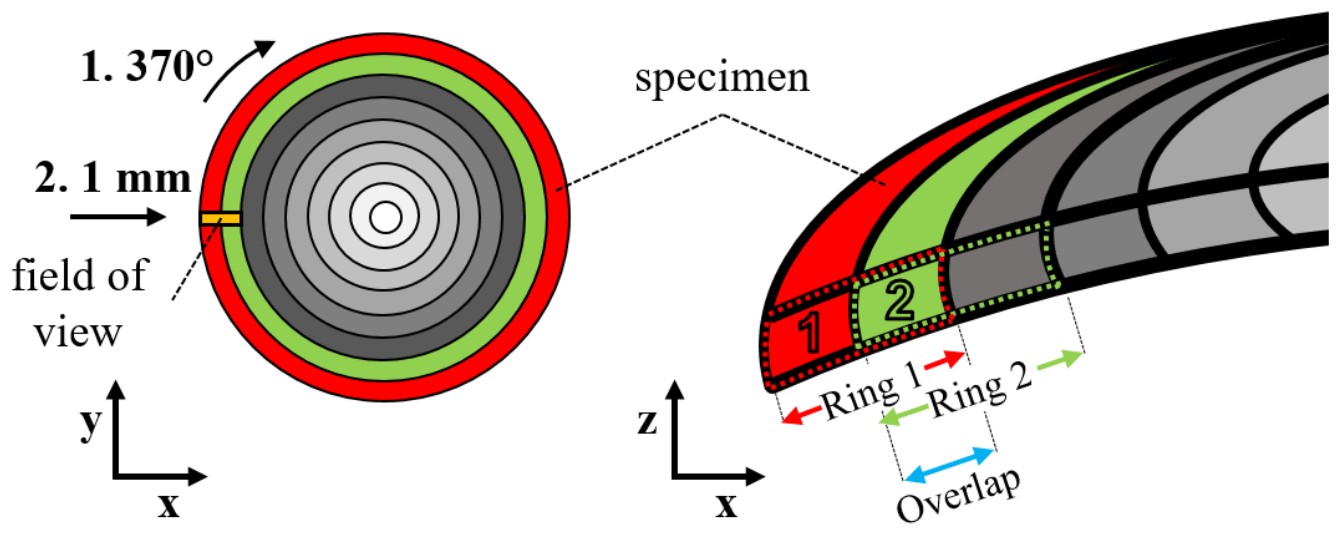

Figure 2: Measurement strategy of OPLM-mode of the sensor. Several ring-shaped sub-apertures are measured subsequently with an overlap. The reconstruction of the complete topography of the specimen is done by stitching of sub-apertures. The overlapping areas are used to correct errors of the movement system.

\section{MEASUREMENT RESULTS}

A result of a high-speed SWLI measurement of a $90 \mathrm{~nm}$ height-step structure with a spatial period of $800 \mu \mathrm{m}$ using a $\mathrm{x}_{\mathrm{b}}$-axis sampling interval of $55 \mathrm{~nm}$ and a scanning speed of $0.5 \mathrm{~mm} / \mathrm{s}$ is depicted in Figure 3. The left part shows the correlogram (black) of one pixel of the measurement including its envelope (blue) and a fitted Gaussian curve (red). The right part presents one period of the evaluated surface profile resulting from the envelope evaluation (blue) and the phase evaluation (red) with the mean height levels (green). The mean absolute height of the detected structure is calculated and used to axially position the sensor in its working distance to that height. For surfaces without height steps, the slope of a linear fit to the measurement data can be used to reduce the tilt of the sensor to the local surface of the specimen until the optical axis of the sensor agrees with the surface normal. 

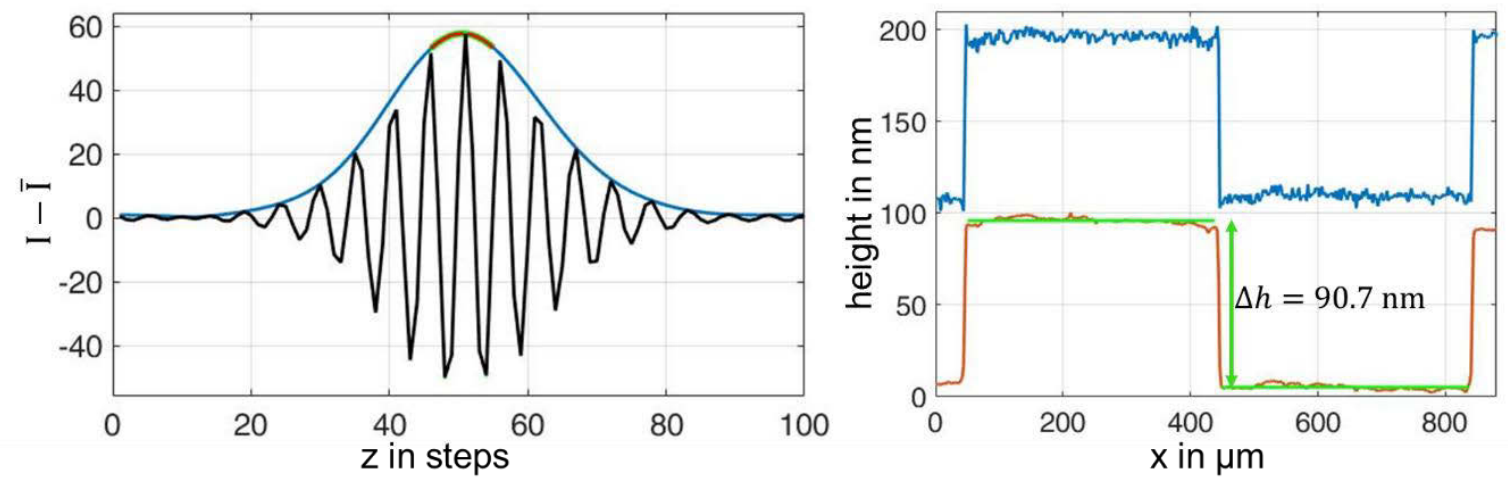

Figure 3: Measurement results of a high-speed white-light measurement of a $800 \mu \mathrm{m}$ periodic, $90 \mathrm{~nm}$ silicon height-step structure; left: band-pass filtered correlogram (black) of one pixel with envelope (blue); right: one period of the evaluated surface profile using envelope-(blue) and phase-evaluation (red) with marked mean height levels (green).

Figure 4 is showing the stitching procedure for a measurement of a sphere with a radius of 200 $\mathrm{mm}$. Figure 4 (a) presents six stitched rings with marked edges between the single rings (black). Figure 4 (b) shows the whole stitched 3D topography. Its deviation from a best-fit sphere results from Figure 4 (c). This picture reveals a systematic height step of approximately $100 \mathrm{~nm}$ at a certain angular position. This is a consequence of the current data correction and stitching strategy which needs to be eliminated by improving the evaluation algorithm. It seems that the best-fit for the stitching in the overlapping area from ring to ring is weighted more than the overlapping area that is measured by one ring scan; however, this is under investigation. In order to check the repeatability of the OPLM-mode of the sensor, a spherical specimen with a

(a)

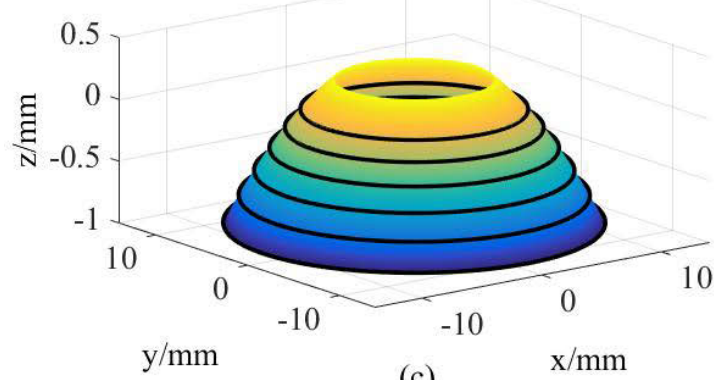

(c)

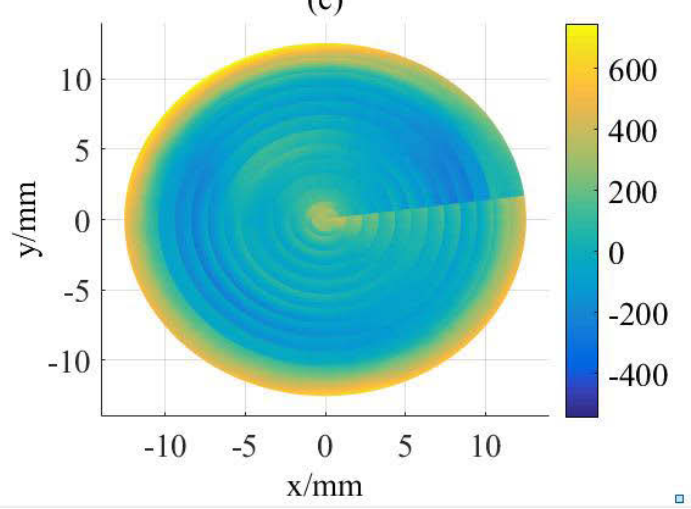

(b)

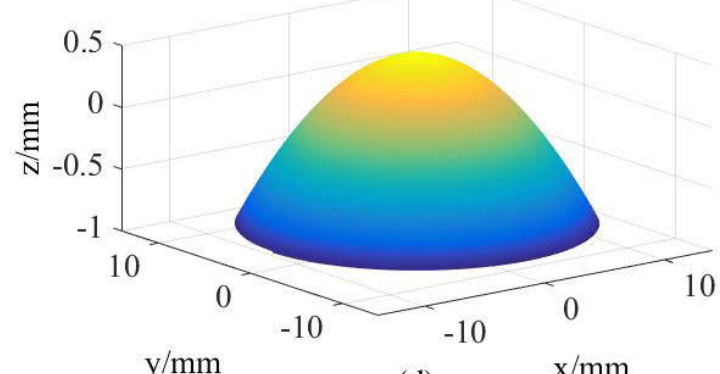

(d)

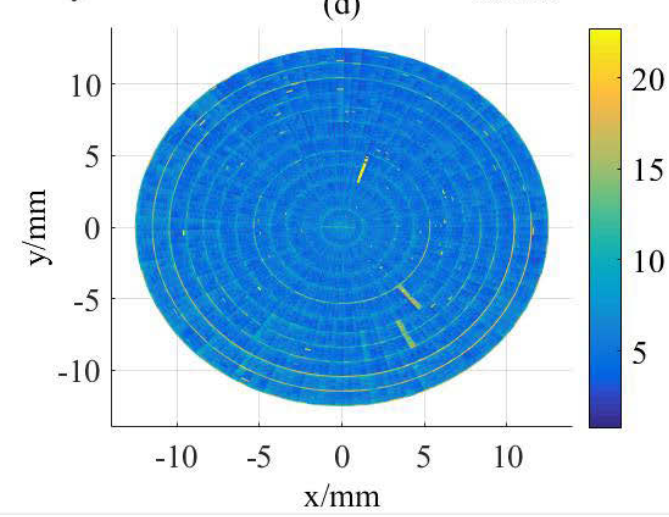

Figure 4: Result of a measurement of a sphere with a radius of $200 \mathrm{~mm}$, (a) - (b) are showing the stitching procedure: (a) six stitched sub-apertures with marked edges (black) are shown; (b) depicts the complete stitched $3 D$ topography; (c) shows the deviation of the reconstructed topography from a best-fit sphere; (d) repeatability of ten 3D topography measurements of a spherical specimen using the stitching algorithm. 
diameter of $25.4 \mathrm{~mm}$ is measured ten times. The measured height data of the single sub-aperture rings are stitched together to a 3D topography. Following this, the median topography and the standard deviation of the ten successively measured topography data sets is calculated. The result is shown in Figure 4 (d), the standard deviation from the median topography, defined as repeatability, is about $5 \mathrm{~nm}$.

\section{CONCLUSION AND OUTLOOK}

The presented interferometric sensor is capable to measure many differently shaped specimen geometries e.g. plane objects, spheres or aspheres with a repeatability in the lower nanometer range. The combination of sinusoidal phase-shifting interferometry for topography measurement with white-light interferometry for accurate positioning provides highly accurate geometry tracking as well as fast and adaptive measurements. Due to its moderate components costs, the system may target the interests of industrial precision measurement and engineering. If higher lateral resolution or a smaller measurable radius of curvature of the specimen is required, the system may easily be adapted by substitution of the used microscope objective lens or by reduction of the length of the line-cameras sensor that is evaluated. By evaluating a single camera pixel, the sensor is able to work as a point sensor as well, including the already presented disadvantages. Given that the current evaluation method takes most of the time of a measurement, the current work includes the implementation of an online evaluation, using commonly available computer hardware. If the height information is available during the measurement, it could be used to track the sensor online to the specimen's surface while rotating. This allows the sensor to measure samples without the need of an accurate manual alignment of the sample to the center of rotation of the c-axis, maybe even to measure freeform surfaces. Further possible improvements include additional sensors that can be used to measure movement errors of the rotational axis c, allowing this axis to be replaced by a more cost-efficient version. A new movement system may also be implemented to measure specimens with diameters of up to $300 \mathrm{~mm}$ and to obtain more redundant data by measuring the complete contour of the specimen, using positive and negative polar angles. 


\section{REFERENCES}

[1] Malacara, D., "Optical Shop Testing," Wiley-Interscience John Wiley \& Sons (2007).

[2] Poleschchuk, A.G. et al., "Combined computer-generated hologram for testing steep aspheric surfaces," Optics Express, 17:5420-5425 (2009).

[3] Malacara, D. et al., "Interferogram Analysis for Optical Testing," Taylor \& Francis (2005).

[4] De Groot, P., "Principles of interference microscopy for the measurement of surface topography," Advances in Optics and Photonics, 7:1-65 (2015).

[5] Millerd, J. et al., "Pixelated Phase-Mask Dynamic Interferometer," Proc. of SPIE, 5531:304-314 (2004).

[6] Donohue, S. et al., "Stitching interferometry for flexible asphere metrology," ASPE Proceedings (2006)

[7] Knell, H. et al., "Continuous measurement of optical surfaces using a line-scan interferometer with sinusoidal path length modulation," Optics Express, 22:2978729789 (2014).

[8] Laubach, S. et al., "A new form measurement system based on subaperture stitching with a line-scanning interferometer," Advanced Optical Technologies, 5:415-422 (2016).

[9] Laubach, S. et al., "Interferometrischer Liniensensor zur Formmessung von rotationssymmetrischen optisch glatten Oberflächen," Technisches Messen, 84:166-173 (2017).

[10] Sasaki, O. et al., "Sinusoidal phase modulating interferometry for surface profile measurement," Applied Optics, 25:3137-3140 (1986).

[11] Sasaki, O. et al., "Analysis of measurement accuracy in sinusoidal phase modulating interferometry," Applied Optics, 25:3152-3158 (1986).

[12] Laubach, S. et al., "Stitching streifenförmiger Subaperturen zur Formmessung," DGaO Proceedings, A20 (2014).

[13] Laubach, S. et al., "Calibration strategies for a new fast line-based form measuring system," DGaO Proceedings, P5 (2015).

\section{CONTACTS}

M.Sc. Jörg Riebeling

Prof. Dr.-Ing. habil. Peter Lehmann

Dipl.-Ing. Sören Laubach

Dr. Gerd Ehret joerg.riebeling@uni-kassel.de

p.lehmann@uni-kassel.de

soeren.laubach@ptb.de

gerd.ehret@ptb.de 